\title{
Europäische und DGN-Leitlinien gehen weitgehend konform
}

Fragestellung: Wie werden Patienten mit Aneurysmen und/ oder Subarachnoidalblutungen am besten diagnostiziert und behandelt?

Hintergrund: Subarachnoidalblutungen haben eine Inzidenz von etwa neun von 100.000. Trotz optimaler neuer Therapieansätze beträgt die Sterblichkeit der Subarachnoidalblutungen immer noch zwischen $50 \%$ und $60 \%$.

Methodik: Eine Arbeitsgruppe aus Neurologen, Neuroradiologen und Neurochirurgen hat eine konsensusbasierte Leitlinie zum Management intrakranieller Aneurysmen und Subarachnoidalblutungen verfasst. Im Folgenden sollen die wichtigsten Empfehlungen wiedergegeben werden.

Ergebnisse: Die wichtigsten Risikofaktoren für Subarachnoidalblutungen, abgesehen vom Alter, sind arterielle Hypertonie, Rauchen und Alkoholmiss-

Steiner T, Juvela S, Unterberg A et al. European stroke organization guidelines for the management of intracranial aneurysms and subarachnoid haemorrhage. Cerebrovasc Dis 2013; 35: $93-112$ brauch. Kommt es bei zwei oder mehr Blutsverwandten zu einer Subarachnoidalblutung ist ein Screening mithilfe der CT- oder MR-Angiografie gerechtfertigt. Im Akutmanagement der Subarachnoidalblutung sollten
Patienten zunächst immobilisiert werden und mit Antiemetika, Analgetika und Laxanzien behandelt werden. Hyperglykämien von über $10 \mathrm{mmol} / \mathrm{l}$ sollten behandelt werden. Dies gilt auch für erhöhte Körpertemperatur. Bis zur Ausschaltung des Aneurysmas sollte der systolische Blutdruck unter $180 \mathrm{mmHg}$ gehalten werden. Bei Patienten mit Subarachnoidalblutung die bettlägerig sind, sollten Kompressionsstrümpfe zur Verhinderung tiefer Beinvenenthrombosen angewendet werden. Bezüglich der spezifischen Therapie sollten Patienten mit Subarachnoidalblutungen so schnell wie möglich operativ oder interventionell behandelt werden. Dabei sollte der optimale Therapieansatz zwischen Neuroradiologen, Neurochirurgen und Neurologen diskutiert werden. Die bevorzugte Therapie ist das Coiling. Faktoren die für eine operative Intervention sprechen sind jüngeres Lebensalter, Raumforderung der intrakraniellen Blutung, Aneurysmen der Arteria cerebri media oder der Arteria pericallosa und Abgang von Arterien direkt aus dem Aneurysma. Bezüglich angiospastischer Insulte sollten Patienten oral mit Nimodipin 60 mg alle vier Stunden behandelt werden. Magnesium ist nicht wirksam. Bei perimesencephalen Subarachnoidalblutungen ist in aller Regel eine CT-Angiografie ausreichend.

Schlussfolgerungen: Die ESO-Leitlinien zur Diagnostik und Therapie von asymptomatischen Aneurysmen und Subarachnoidalblutungen stimmen weitestgehend mit denen der DGN überein.

\section{-Kommentar von Hans-Christoph Diener, Essen}

\section{Wertvolle Hilfen im Alltag}

Diese von der Europäischen Schlaganfallorganisation erarbeiteten und veröffentlichten Leitlinien zur Diagnostik und Therapie von Subarachnoidalblutungen sind eine wertvolle Hilfe für den klinischen Alltag. Mit Ausnahme der randomisierten Studien zum Coiling und Clipping von Aneurysmen gibt es allerdings relativ wenige randomisierte Therapiestudien, auf die sich die Therapieempfehlungen stützen können. Studien gibt

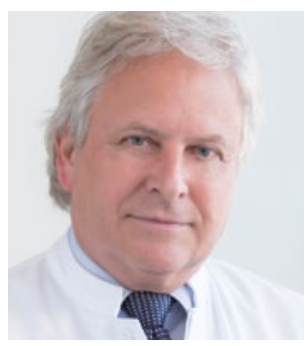

Prof. Dr. med. Hans-Christoph, Diener, Essen

Direktor der Klinik für Neurologie, Universitätsklinikum Essen E-Mail:h.diener@uni-essen.de

es im medikamentösen Bereich bisher nur für Nimodipin. Alle anderen Neuroprotektiva waren nicht wirksam. Erfreulicherweise stimmen die Europäischen Leitlinien weitestgehend mit denen der DGN überein.

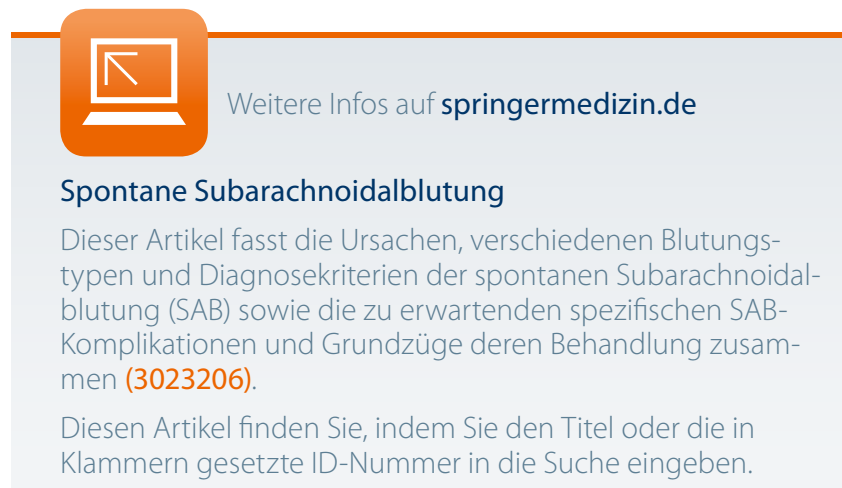

